\title{
Retiming Synchronous Circuitry ${ }^{1}$
}

\author{
Charles E. Leiserson ${ }^{2}$ and James B. Saxe ${ }^{3}$
}

\begin{abstract}
This paper describes a circuit transformation called retiming in which registers are added at some points in a circuit and removed from others in such a way that the functional behavior of the circuit as a whole is preserved. We show that retiming can be used to transform a given synchronous circuit into a more efficient circuit under a variety of different cost criteria. We model a circuit as a graph in which the vertex set $V$ is a collection of combinational logic elements and the edge set $E$ is the set of interconnections, each of which may pass through zero or more registers. We give an $O(|V||E| \lg |V|)$ algorithm for determining an equivalent retimed circuit with the smallest possible clock period. We show that the problem of determining an equivalent retimed circuit with minimum state (total number of registers) is polynomial-time solvable. This result yields a polynomial-time optimal solution to the problem of pipelining combinational circuitry with minimum register cost. We also give a chacterization of optimal retiming based on an efficiently solvable mixed-integer linear-programming problem.
\end{abstract}

Key Words. Digital circuitry, Graph theory, Linear programming, Network flow, Optimization, Pipelining, Propagation delay, Retiming, Synchronous circuitry, Systolic circuits, Timing analysis.

1. Introduction. The goal of VLSI design automation is to speed the design of a system without sacrificing the quality of implementation. A common means of achieving this goal is through the use of optimization tools that improve the quality of a quickly designed circuit. In this paper we show how to optimize clocked circuits by relocating registers so as to reduce combinational rippling. Unlike pipelining, this technique, which we call retiming, does not increase circuit latency.

In order to illustrate retiming, consider the problem of designing a digital correlator. The correlator takes a stream of bits $x_{0}, x_{1}, x_{2}, \ldots$ as input and compares it with a fixed-length pattern $a_{0}, a_{1}, \ldots, a_{k}$. After receiving each input $x_{i}$ $(i \geq k)$, the correlator produces as output the number of matches

$$
y_{i}=\sum_{j=0}^{k} \delta\left(x_{i-j}, a_{j}\right)
$$

\footnotetext{
${ }^{1}$ This research was supported in part by the Defense Advanced Research Projects Agency under Contract N00014-80-C-0622 and by the Office of Naval Research under Contract N00014-76-C-0370. Charles Leiserson was supported in part by an NSF Presidential Young Investigator Award with matching funds provided by AT\&T Corporation, IBM Corporation, and Xerox Corporation. Most of the results reported here were obtained while James Saxe was in the Computer Science Department at Carnegie-Mellon University. During part of that period, he was supported by an IBM graduate fellowship.

${ }^{2}$ Laboratory for Computer Science, Massachusetts Institute of Technology, Cambridge, MA 02139, USA.

${ }^{3}$ DEC Systems Research Center, 130 Lytton Avenue, Palo Alto, CA 94301, USA.
}

Received October, 1986; revised September 29, 1988. Communicated by F. Thomson Leighton. 


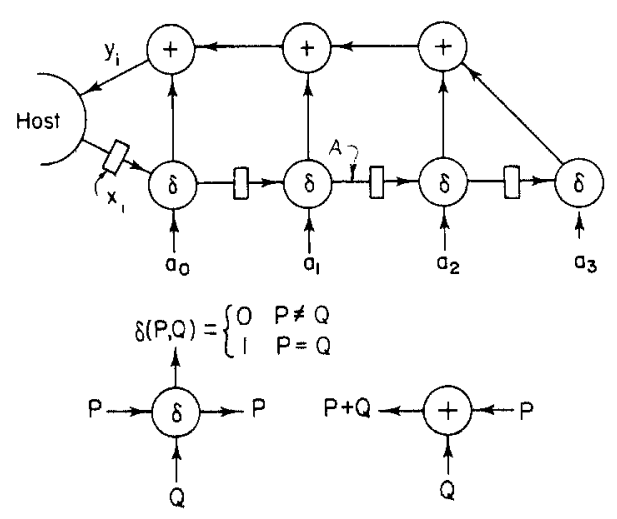

Fig. 1. Correlator 1: a simple circuit made of two kinds of functional elements. Each comparator $\delta$ has a propagation delay of $3 \mathrm{esec}$, and each adder + has a propagation delay of 7 esec. A longest path of combinational rippling starts at the register on the connection labeled $A$, and thus the clock period of the circuit is 24 esec.

where $\delta$ is the comparison function

$$
\delta(x, y)= \begin{cases}1 & \text { if } x=y \\ 0 & \text { otherwise }\end{cases}
$$

Figure 1 shows a design of a simple correlator for the case when $k=3$. Correlator 1 consists of two kinds of functional elements, adders and comparators, whose I/O characteristics are shown in the figure. The boxes between the comparators are registers which act to shift the $x_{i}$ to the right down the length of the correlator. On each tick of the global clock, each $x_{i}$ is compared with a character of the pattern, and the adders sum up the number of matches.

This design, though easy to understand, has poor performance. Between ticks of the clock, the partial sums of the matches ripple up the length of the correlator. Suppose, for instance, that each adder has a propagation delay of $7 \mathrm{esec},{ }^{4}$ and each comparator has a propagation delay of 3 esec. Then the clock period must be at least 24 esec-the time for a signal to propagate from the register on the connection labeled $A$ through one comparator and three adders.

A design that gives better performance can be derived by removing the register on connection $A$ from Correlator 1 and inserting a new register on connection $B$, as shown in Figure 2. To show that these two correlators are indeed functionally equivalent, consider the portion of the circuit surrounded by the dashed box in the figure. It communicates with the rest of the circuit only through connections $A$ and $B$. When the register on $A$ is removed, all input signals to this portion of the circuit arrive one clock tick earlier, and thus the boxed portion of Correlator 2 performs the same sequence of computations as in Correlator 1 , but one clock tick earlier. Since the output from the boxed portion of Correlator 2 is delayed one clock tick by the new register on connection $B$, the remainder of the circuit sees the same

\footnotetext{
${ }^{4}$ Recall that one eptosecond (esec) equals one one-zillionth of a second.
} 


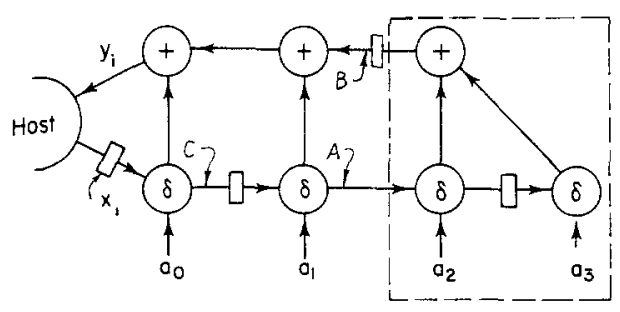

Fig. 2. Correlator 2: a retimed circuit functionally equivalent to, but more efficient than, Correlator 1 . The longest path of combinational rippling begins at the register on connection $C$, and the clock period of this circuit is 17 esec.

behavior as in Correlator 1. We say that the three functional elements in the boxed portion of Correlator 2 lead by one clock tick the corresponding functional elements in Correlator 1. Alternatively, we say that the three elements in Correlator $1 \mathrm{lag}$ by one clock tick the corresponding elements in Correlator 2 .

Correlators 1 and 2 are functionally equivalent, but the performance of the retimed circuit Correlator 2 is better than that of Correlator 1 . The clock period of Correlator 2 is 17 esec-the time for a signal to propagate from the register on connection $C$ through one comparator and two adders. Notice that the two designs use the same functional elements connected in the same manner and differ only in the locations of registers. Correlator 2 has the I/O characteristic specified by (1), but it should be apparent that a direct verification requires considerably more effort than the verification of Correlator 1.

Retiming, the technique of inserting and deleting registers in such a way as to preserve function, can be used to produce an even faster circuit than Correlator 2. Section 4 gives an implementation of the correlator that achieves a clock period of 13 esec. Remarkably, if the pattern of comparators and adders is extended arbitrarily to the right, a clock period of 14 esec can always be achieved by retiming. In this paper we exhibit a polynomial-time algorithm for determining a retiming of a circuit that minimizes clock period.

The remainder of this paper is organized as follows. Section 2 presents the graphtheoretic model of synchronous circuits used in this paper. In Section 3 we formally describe the operation of retiming [14], in which registers are deleted from some connections of a circuit and added to others so that the circuit function is preserved. Section 4 gives a simple polynomial-time algorithm for minimizing the clock period of a circuit. Section 5 gives an asymptotically more efficient algorithm to solve the same problem. In Section 6 we show that the problem of finding a retiming of a circuit that minimizes clock period can be reduced to an efficiently solvable mixed-integer linear-programming problem, thus providing a framework for retiming based on mathematical programming.

Sections 7-9 discuss extensions of these results. Section 7 considers the special case where all functional elements have identical propagation delays and shows that optimal retimings can be found more efficiently in this case. The section also discusses the relationship of this work to systolic computation and shows how to improve the performance of many systolic circuits in the literature. While earlier 
sections are concerned with finding retimings that are optimal in the sense of minimizing clock period, Section 8 examines a different optimization criterion, namely minimizing the total amount of state (number of registers) in the retimed circuit. In particular, we show that the problem of retiming a circuit to minimize its state subject to an upper bound on the clock period can be reduced to the linearprogramming dual of a minimum-cost flow problem, and hence can be solved optimally in polynomial time. Section 9 extends our methods to a more general circuit model in which individual functional elements may have nonuniform propagation delays-e.g., the low-order output bit of an adder may be available earlier than the high-order bit.

In Section 10 we briefly mention further extensions, including the application of our algorithms to optimal pipelining of combinational circuitry.

2. Preliminaries. In this section we define the notations and terminology needed in this paper and present our graph-theoretic model of digital circuits. We conclude by giving a simple algorithm for determining the minimum feasible clock period of a circuit from its graph.

We can view a circuit abstractly as a network of functional elements and globally clocked registers. The registers are assumed to have the following characteristics: each has a single input and a single output; all are clocked by the same periodic waveform; and at each clock tick, each storage element samples its input and the sampled value is made available at the output until the next tick. We also assume that changes in the output of one storage element do not interfere with the input to another at the same clock tick. An example of such a storage element is an edgetriggered, master-slave, D-type flip-flop [21].

The functional elements provide the computational power of the circuit. Our model is unconcerned with the level of complexity of the the functional elements-they might be NAND gates, multiplexors, or ALUs, for example. Each functional element has an associated propagation delay. The outputs of a functional element at any time are defined as a specified function of its inputs, provided that all the inputs have been stable for a time at least equal to the element's propagation delay. We make the conservative assumption that when an input to a functional element changes, the outputs may behave arbitrarily until they settle to their final values.

To be precise, we model a circuit as a finite, vertex-weighted, edge-weighted, directed multigraph $G=\langle V, E, d, w\rangle$ (henceforth, we simply say "graph" or, more frequently, "circuit"). Figure 3 shows the graph of Correlator 1 from Figure 1. The

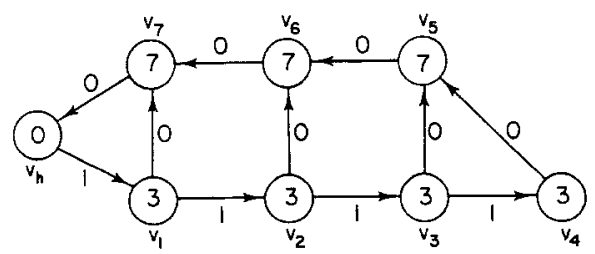

Fig. 3. The graph model of Correlator 1 from Figure 1 . 
vertices $V$ of the graph model the functional elements of the circuit. Each vertex $v \in V$ is weighted with its numerical propagation delay $d(v)$. The directed edges $E$ of the graph model interconnections between functional elements. Each edge $e \in E$ connects an output of some functional element to an input of some functional element and is weighted with a register count $w(e)$. The register count is the number of registers along the connection. ${ }^{5}$ Between two vertices, there may be multiple edges with different register counts.

Vertices can be designated to represent interfaces with the external world, and each such vertex is given zero propagation delay, as is shown for vertex $v_{h}$ in Figure 3. (We elaborate on this technicality in Section 10.) If the relative times of events at multiple external interfaces must be preserved, we treat them as a single interface and represent them as a single vertex with multiple incident edges. Otherwise, we assume that multiple external interfaces are independent of each other and cannot communicate with each other externally. For most of our theory, external interfaces can be handled as ordinary vertices, and thus our formalism omits them.

We use the following terminology extensively. To avoid confusion between vertex-weight functions such as the propagation delay $d$ and edge-weight functions such as the register count $w$, we use the term weight for edge-weight functions only. In fact, the only vertex-weight functions we use are the propagation delays $d(v)$, and in general we refer to the particular edge weights $w(e)$ of a circuit as register counts. If $e$ is an edge in a graph that goes from vertex $u$ to vertex $v$, we use the notation $u \stackrel{e}{\rightarrow} v$. In the event that the identity of either the head or the tail of an edge is unimportant, we use the symbol?, as in $u \stackrel{e}{\rightarrow}$ ?.

For a graph $G$, we view a path $p$ in $G$ as a sequence of vertices and edges. If a path $p$ starts at a vertex $u$ and ends at a vertex $v$, we use the notation $u \stackrel{p}{\rightarrow} v$. A simple path contains no vertex twice, and therefore the number of vertices exceeds the number of edges by exactly one.

We extend the register count function $w$ in a natural way from single edges to arbitrary paths. For any path $p=v_{0} \stackrel{e_{\rightarrow}}{\rightarrow} v_{1} \stackrel{e_{1}}{\rightarrow} \ldots \stackrel{e_{k-1}}{\rightarrow} v_{k}$, we define the path weight as the sum of the weights of the edges of the path:

$$
w(p)=\sum_{i=0}^{k-1} w\left(e_{i}\right)
$$

Similarly, we extend the propagation delay function $d$ to simple paths. For any simple path $p=v_{0} \stackrel{e_{0}}{\rightarrow} v_{1} \stackrel{e_{1}}{\rightarrow} \cdots \stackrel{e_{k-1}}{\longrightarrow} v_{k}$, we define the path delay as the sum of the delays of the vertices of the path:

$$
d(p)=\sum_{i=0}^{k} d\left(v_{i}\right)
$$

\footnotetext{
${ }^{5}$ If an output of a functional element fans out to more than one other functional element, the single interconnection can be treated, without loss of generality, as several edges, each with an appropriate weight. Any optimization can be translated from the model back to a circuit with fanout. Section 8 examines fanout more closely.
} 
In order that a graph $G=\langle V, E, d, w\rangle$ have well-defined physical meaning as a circuit, we place nonnegativity restrictions on the propagation delays $d(v)$ and the register counts $w(e)$ :

D1. The propagation delay $d(v)$ is nonnegative for each vertex $v \in V$.

W1. The register count $w(e)$ is a nonnegative integer for each edge $e \in E$.

We also impose the restriction that there be no directed cycles of zero weight:

W2. In any directed cycle of $G$, there is some edge with (strictly) positive register count.

We define a synchronous circuit as a circuit that satisfies conditions D1, W1, and $\mathrm{W} 2$. The reason for including condition W2 is that whenever an edge $e$ between two vertices $u$ and $v$ has zero weight, a signal entering vertex $u$ can ripple unhindered through vertex $u$ and subsequently through vertex $v$. If the rippling can feed back upon itself, problems of asynchronous latching, oscillation, and race conditions can arise. By prohibiting zero-weight cycles, condition W2 prevents these problems from occurring, provided that the system clock runs slowly enough to allow the outputs of all the functional elements to settle between each two consecutive ticks.

For any synchronous circuit $G$, we define the (minimum feasible) clock period $\Phi(G)$ as the maximum amount of propagation delay through which any signal must ripple between clock ticks. Condition W2 guarantees that the clock period is well defined by the equation

$$
\Phi(G)=\max \{d(p): w(p)=0\} .
$$

For the circuit graph in Figure 3 the clock period is 24, which corresponds to the sum of the propagation delays along the path $v_{4} \rightarrow v_{5} \rightarrow v_{6} \rightarrow v_{7}$.

Determination of the clock period $\Phi(G)$ is relatively simple. The algorithm we present here is similar to an algorithm that forms a part of a design tool developed at American Microsystems, Inc. [17].

Algorithm CP (compute the clock period of a circuit). This algorithm computes the clock period $\Phi(G)$ for a synchronous circuit $G=\langle V, E, d, w\rangle$.

1. Let $G_{0}$ be the subgraph of $G$ that contains precisely those edges $e$ with register count $w(e)=0$.

2. By condition W2, $G_{0}$ is acyclic. Perform a topological sort on $G_{0}$, totally ordering its vertices so that if there is an edge from vertex $u$ to vertex $v$ in $G_{0}$, then $u$ precedes $v$ in the total order.

3. Go through the vertices in the order defined by the topological sort. On visiting each vertex $v$, compute the quantity $\Delta(v)$ as follows:

a. If there is no incoming edge to $v$, set $\Delta(v) \leftarrow d(v)$.

b. Otherwise, set $\Delta(v) \leftarrow d(v)+\max \{\Delta(u): u \stackrel{e}{\rightarrow} v$ and $w(e)=0\}$.

4. The clock period $\Phi(G)$ is $\max _{v \in V} \Delta(v)$. 
The algorithm works because, for each vertex $v$, the quantity $\Delta(v)$ equals the maximum sum $d(p)$ of vertex delays along any zero-weight directed path $p$ in $G$ such that $\stackrel{\stackrel{p}{\rightarrow}}{\rightarrow} v$. The running time is $O(|E|)$.

3. Retiming. Retiming transformations alter the clock period of a circuit by inserting and deleting registers, but without otherwise affecting the circuit's structure. This section formally defines retiming and proves some simple properties of the transformation.

A retiming can be viewed as an assignment of a lag to each vertex in a circuit, and this is how we define it formally. A retiming of a circuit $G=\langle V, E, d, w\rangle$ is an integer-valued vertex-labeling $r: V \rightarrow \mathbf{Z}$. The retiming specifies a transformation of the original circuit in which registers are added and removed so as to change the graph $G$ into a new graph $G_{r}=\left\langle V, E, d, w_{r}\right\rangle$, where the edge-weighting $w_{r}$ is defined for an edge $u \stackrel{e}{\rightarrow} \mathrm{v}$ by the equation

$$
w_{r}(e)=w(e)+r(v)-r(u) .
$$

In the example of Figure 3, the retiming that assigns -1 to functional elements $v_{3}, v_{4}$, and $v_{5}$, and assigns 0 to all other vertices, yields the circuit of Figure 2.

Equation (2), which tells how retiming affects the register counts of edges, extends naturally to paths.

LEMMA 1. Let $G=\langle V, E, d, w\rangle$ be a synchronous circuit, and let $r$ be a retiming. Then for any path $u \stackrel{p}{\rightarrow} v$ in $G$, we have

$$
w_{r}(p)=w(p)+r(v)-r(u) .
$$

Proof. Suppose $p$ is composed of vertices and edges $v_{0} \stackrel{e_{0}}{\rightarrow} v_{1} \stackrel{e_{1}}{\rightarrow} \cdots \stackrel{e_{k-1}}{\rightarrow} v_{k}$. We have

$$
\begin{aligned}
w_{r}(p) & =\sum_{i=0}^{k-1} w_{r}\left(e_{i}\right) \\
& =\sum_{i=0}^{k-1}\left(w\left(e_{i}\right)+r\left(v_{i+1}\right)-r\left(v_{i}\right)\right) \\
& =\sum_{i=0}^{k-1} w\left(e_{i}\right)+\sum_{i=0}^{k-1}\left(r\left(v_{i+1}\right)-r\left(v_{i}\right)\right) \\
& =w(p)+r\left(v_{k}\right)-r\left(v_{0}\right)
\end{aligned}
$$

because the sum on the right telescopes.

COROllaRy 2. Let $G=\langle V, E, d, w\rangle$ be a synchronous circuit, and let $r$ be a retiming on the vertices of $G$. Then, for any cycle $p$ in $G$, we have $w_{r}(p)=w(p)$.

Proof. Immediate from Lemma 1. 
A retiming $r$ of a circuit $G$ is legal if the retimed graph $G_{r}$ satisfies conditions W1 and W2. An arbitrary assignment of lags to the vertices of a circuit $G$ may cause the retimed circuit $G_{r}$ to violate condition W1, which says that no edge may have a negative register count. This condition must be checked explicitly in order to ensure that a retiming is legal. Interestingly enough, condition W2 need not also be checked because of the following consequence of Corollary 2 .

COROLlary 3. Let $G=\langle V, E, d, w\rangle$ be a synchronous circuit, and let $r$ be a retiming on the vertices of $G$ such that $G_{r}$ satisfies condition $W 1$. Then $r$ is a legal retiming.

Proof. Since the propagation delays $d$ are unaffected by retiming, condition D1 is satisfied by graph $G_{r}$. Since condition W1 is true by supposition, it remains only to show that $G_{r}$ satisfies condition W2. Let $p$ be any cycle in $G$. We must show that $p$ includes at least one edge $e$ such that $w_{r}(e)>0$. Since graph $G$ satisfies conditions $\mathrm{W} 1$ and W2, the register count $w(p)$ of the cycle in $G$ must be positive. But by Corollary 2, the register count $w_{r}(p)$ of the cycle in $G_{r}$ is equal to $w(p)$ and is therefore positive. Hence, there must be an edge on the cycle in $G_{r}$ that has positive register count.

To conclude this section we comment that it is necessary to prove that when a circuit $G$ is retimed to produce a new graph $G_{r}$, the new circuit is functionally equivalent, as seen by the external world, to the original-provided, of course, that $G_{r}$ satisfies conditions W1 and W2. Such a proof can be found in [14], which also contains a technical definition of the term "equivalent."

Moreover, we can show that retiming is, in a sense, the most general possible method for changing the register counts within a circuit without disturbing the circuit's function. Although we do not formally prove it here, we outline the thread of reasoning. (For an example of a similar argument used to prove a weaker result, see the proof of Theorem 3 in [14].) Without loss of generality, assume that any circuit $G=\langle V, E, d, w\rangle$ under discussion has the following two properties.

1. Every vertex $v \in V$ is connected by a path to some external interface.

2. Every vertex $v \in V$ has at least one input. (Otherwise, $v$ computes a constant function. ${ }^{6}$ )

Given the graph of such a circuit, but no knowledge of what functions are computed by the functional elements, it is impossible, other than by retiming, to alter the register counts on the edges and be assured that the external behavior is unchanged. For any relabeling of the edge weights that is not a retiming, an adversary can specify the functional elements in such a way that the new circuit behaves differently from the original circuit. We omit the details of this argument.

\footnotetext{
${ }^{6}$ In the graph (Figure 1) of Correlator 1, for example, we do not use functional elements to input the constants $a_{\mathrm{i}}$, but have instead incorporated them into the comparators.
} 
4. An Algorithm for Clock Period Minimization. This section presents a polynomial-time algorithm for retiming a circuit so as to maximize performance. Specifically, we solve the following clock-period-minimization problem: Given a circuit graph $G=\langle V, E, d, w\rangle$, find a legal retiming $r$ of $G$ such that the clock period $\Phi\left(G_{r}\right)$ of the retimed circuit $G_{r}$ is as small as possible. The solution of this problem depends on some basic results from combinatorial optimization and graph theory. In particular, we rely on the fact that the following linear-programming problem can be solved efficiently.

Problem LP. Let $S$ be a set of $m$ linear inequalities of the form

$$
x_{j}-x_{i} \leq a_{i j}
$$

on the unknowns $x_{1}, x_{2}, \ldots, x_{n}$, where the $a_{i j}$ are given real constants. Determine feasible values for the unknowns $x_{i}$, or determine that no such values exist.

Constraint systems in which each constraint has the form of inequality (3) arise in shortest paths problems and have been studied extensively. Such a system of linear inequalities can be satisfied-or determined to be inconsistent-in $O(m n)$ time by the Bellman-Ford algorithm [10, p. 74].

The algorithm for minimizing the clock period of a circuit is based on an alternative characterization of clock period in terms of two quantities which we now define:

$$
\begin{aligned}
W(u, v) & =\min \{w(p): u \stackrel{p}{\rightarrow} v\}, \\
D(u, v) & =\max \{d(p): u \stackrel{p}{\rightarrow} v \text { and } w(p)=W(u, v)\} .
\end{aligned}
$$

The quantity $W(u, v)$ is the minimum number of registers on any path from vertex $u$ to vertex $v$. We call a path $u \stackrel{p}{\rightarrow} v$ such that $w(p)=W(u, v)$ a critical path from $u$ to $v$. The quantity $D(u, v)$ is the maximum total propagation delay on any critical path from $u$ to $v$. Both quantities are undefined if there is no path from $u$ to $v$. Table 1 shows the values for Correlator 1.

LeMMA 4. Let $G=\langle V, E, d, w\rangle$ be a synchronous circuit, and let $c$ be any positive real number. The following are equivalent:

4.1. $\Phi(G) \leq c$.

4.2. For all vertices $u$ and $v$ in $V$, if $D(u, v)>c$, then $W(u, v) \geq 1$.

Proof. (4.1 $\Rightarrow 4.2)$ Suppose $\Phi(G) \leq c$, and let $u$ and $v$ be vertices in $V$ such that $D(u, v)>c$. If $W(u, v)=0$, then there exists a path $p$ from $u$ to $v$ with propagation delay $d(p)=D(u, v)$, which is greater than $c$, and register count $w(p)=W(u, v)=0$. Contradiction.

$(4.2 \Rightarrow 4.1)$ Suppose 4.2 holds, and let $u \stackrel{p}{\rightarrow} v$ be any zero-weight path in $G$. Then we have $W(u, v)=w(p)=0$, which implies $d(p) \leq D(u, v) \leq c$. 


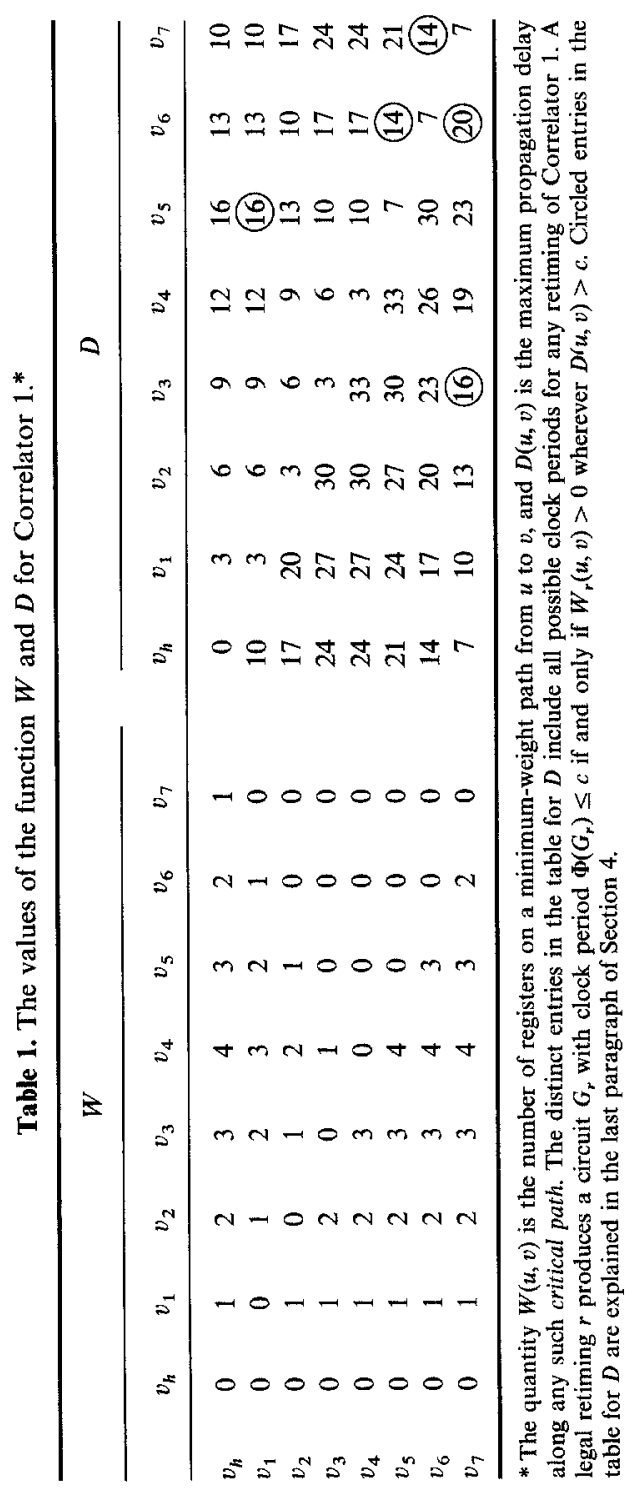


It is not difficult to compute $W$ by solving the all-pairs shortest-paths problem in $G$. Common ways of solving this problem are the Floyd-Warshall method [10, p. 86], which runs in $O\left(|V|^{3}\right)$ time, and Johnson's algorithm [7], which runs in $O\left(|V||E|+|V|^{2} \lg |V|\right)$ time using the Fibonacci heap data structure due to Fredman and Tarjan [2]. The basic operations on weights used by these algorithms are addition and comparison. The following algorithm shows that with a suitably chosen weight function, an all-pairs shortest-paths algorithm can be used to compute both $W$ and $D$.

Algorithm WD (compute $W$ and $D$ ). Given a synchronous circuit $G=$ $\langle V, E, d, w\rangle$, this algorithm computes $W(u, v)$ and $D(u, v)$ for all $u, v \in V$ such that $u$ is connected to $v$ in $G$.

1. Weight each edge $u \stackrel{e}{\rightarrow}$ ? in $E$ with the ordered pair $(w(e),-d(u))$.

2. Using the weighting from step 1, compute the weight of the shortest path joining each connected pair of vertices by solving an all-pairs shortest-paths algorithm. (In the all-pairs algorithm, add two weights by performing componentwise addition. Compare weights using lexicographic ordering.)

3. For each shortest-path weight $(x, y)$ between two vertices $u$ and $v$, set $W(u, v) \leftarrow$ $x$ and $D(u, v) \leftarrow d(v)-y$.

The reason that $W$ and $D$ are important is that they behave nicely under retiming.

Lemma 5. Let $G=\langle V, E, d, w\rangle$ be a synchronous circuit, and let $W$ and $D$ be defined on $G$ by (4). Let $r$ be a legal retiming of $G$, and let $W_{r}$ and $D_{r}$ be defined analogously on $G_{r}$. Then

5.1. a path $p$ is a critical path of $G_{r}$ if and only if it is a critical path of $G$,

5.2. $W_{r}(u, v)=W(u, v)+r(v)-r(u)$ for all connected vertices $u, v \in V$, and

5.3. $D_{r}(u, v)=D(u, v)$ for all connected vertices $u, v \in V$.

Proof. Condition 5.1 follows from Lemma 1 because retiming changes the weights of all paths from $u$ to $v$ by the same amount, and then 5.2 follows immediately. Condition 5.3 is a consequence of 5.2 together with the fact that retiming does not alter propagation delays.

The next result is a corollary to Lemma 5 which shows that the range of $D$ contains the clock periods of all circuits obtainable by retiming $G$. In Table 1 the 20 distinct values for $D$ include all possible clock periods for any retiming of Correlator 1 .

COROllary 6. Let $G=\langle V, E, d, w\rangle$ be a synchronous circuit, and let $r$ be a retiming of $G$. Then the clock period $\Phi\left(G_{r}\right)$ is equal to $D(u, v)$ for some $u, v \in V$.

Proof. By the definition of clock period, the circuit $G_{r}$ contains some zero-weight path $u \stackrel{p}{\rightarrow} v$ such that $d(p)=\Phi\left(G_{r}\right)$, and thus we have $W_{r}(u, v)=w_{r}(p)=0$. 
Moreover, no zero-weight path in $G_{r}$ has greater propagation delay than $p$, which implies $D_{r}(u, v)=d(p)$. Hence, by Lemma 5 we have $\Phi\left(G_{r}\right)=D_{r}(u, v)=D(u, v)$.

Lemmas 4 and 5 also allow us to characterize the conditions under which a retiming produces a circuit whose clock period is no greater than a given constant.

THEOREM 7. Let $G=\langle V, E, d, w\rangle$ be a synchronous circuit, let $c$ be an arbitrary positive real number, and let $r$ be a function from $V$ to the integers. Then $r$ is a legal retiming of $G$ such that $\Phi\left(G_{r}\right) \leq c$ if and only if

7.1. $r(u)-r(v) \leq w(e)$ for every edge $u \stackrel{e}{\rightarrow} v$ of $G$, and

7.2. $r(u)-r(v) \leq W(u, v)-1$ for all vertices $u, v \in V$ such that $D(u, v)>c$.

Proof. By Corollary 3, the retiming $r$ is legal if and only if condition 7.1 holds. If $r$ is indeed a legal retiming of $G$, then by Lemma 4 the retimed circuit $G_{r}$ has clock period $\Phi\left(G_{r}\right) \leq c$ precisely under the condition that $W_{r}(u, v) \geq 1$ for all vertices $u, v \in V$ such that $D_{r}(u, v)>c$. Since by Lemma 5 we have $W_{r}(u, v)=W(u, v)+$ $r(v)-r(u)$ and $D_{r}(u, v)=D(u, v)$, this condition is equivalent to condition 7.2.

Theorem 7 provides the basic tool needed to solve the clock-period-minimization problem. Notice that the constraints on the unknowns $r(v)$ in the theorem are linear inequalities involving only differences of unknowns, and thus we have an instance of Problem LP. ${ }^{7}$ Therefore, using the Bellman-Ford algorithm to test whether a retimed circuit exists with clock period less than some constant $c$ takes $O\left(|V|^{3}\right)$ time since there can be only $O\left(|V|^{2}\right)$ inequalities.

We now present an algorithm to determine a retiming for a circuit $G$ such that the clock period of the retimed circuit is minimized.

Algorithm OPT1 (clock-period minimization). Given a synchronous circuit $G=$ $\langle V, E, d, w\rangle$, this algorithm determines a retiming $r$ such that $\Phi\left(G_{r}\right)$ is as small as possible.

1. Compute $W$ and $D$ using Algorithm WD.

2. Sort the elements in the range of $D$.

3. Binary search among the elements $D(u, v)$ for the minimum achievable clock period. To test whether each potential clock period $c$ is feasible, apply the Bellman-Ford algorithm to determine whether the conditions in Theorem 7 can be satisfied.

4. For the minimum achievable clock period found in step 3, use the values for the $r(v)$ found by the Bellman-Ford algorithm as the optimal retiming.

Algorithm OPT1 runs in $O\left(|V|^{3} \lg |V|\right)$ time. For some circuits, we can sometimes improve the performance of Algorithm OPT1 by using a smaller set of

\footnotetext{
${ }^{7}$ Actually, we have the integer linear-programming version of the problem because the unknowns $r(v)$ are required to be integer. Since the value on the right-hand side of each equation is integer, however, the Bellman-Ford algorithm produces an integer optimal solution if one exists.
} 


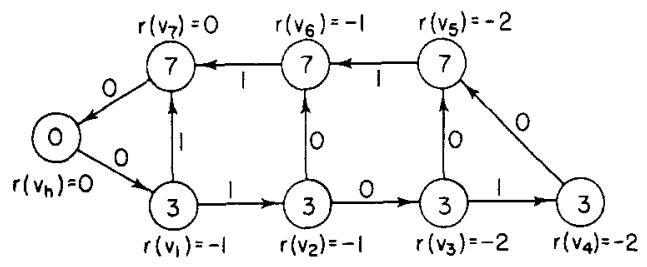

Fig. 4. The graph model of an optimal correlator with clock period 13. The circuit is obtained from the graph of Correlator 1 in Figure 3 by applying the optimal retiming, determined by Algorithm OPT1. For each vertex $v$, the value $r(v)$ is the lag of $v$ with respect to the corresponding vertex in Correlator 1 . The retimed weight of an edge $u \stackrel{e}{\rightarrow} v$ is given by $w_{r}(e)=w(e)+r(v)-r(u)$.

inequalities. (An algorithm with a provably better asymptotic performance is given in the next section.) The key observation is that we may eliminate any inequality $r(u)-r(v) \leq W(u, v)-1$ from condition 7.2 if either $D(u, v)-d(v)>c$ or $D(u, v)-$ $d(u)>c$. The intuition behind this optimization is that there is no need to require explicitly that a path $p$ have positive weight $w_{r}(p)$ if we already require some subpath of $p$ to have positive weight.

As an example, Figure 4 shows the circuit graph of Correlator 3, which can be obtained from Correlator 1 by applying the Bellman-Ford algorithm to the inequalities from Theorem 7 with clock period $c=13$. There are 11 inequalities (one for each edge) that must be satisfied to ensure a legal retiming - condition 7.1 in Theorem 7. Of the potential 34 inequalities arising from cases where $D(u, v)>$ 13 -condition 7.2 in the theorem - only five need be included if we eliminate those for which either $D(u, v)-d(v)>13$ or $D(u, v)-d(u)>13$. Those entries corresponding to the five relevant inequalities for $D$ are circled in Table 1.

5. A More Efficient Algorithm for Clock-Period Minimization. In this section we describe an asymptotically more efficient algorithm for the clock-period-minimization problem. Specifically, we show that the feasible clock-period test in step 3 of Algorithm OPT1, which determines whether there exists a retiming of $G$ with clock period at most $c$, can be performed in $O(|V||E|)$ time, a significant improvement over $O\left(|V|^{3}\right)$ for sparse graphs. This result yields an $O(|V||E| \lg |V|)$-time algorithm for determining the optimal retiming.

We begin with the $O(|V||E|)$-time algorithm for determining whether a given clock period is feasible.

Algorithm FEAS (feasible clock-period test). Given a synchronous circuit $G=$ $\langle V, E, d, w\rangle$ and a desired clock period $c$, this algorithm produces a retiming $r$ of $G$ such that $G_{r}$ is a synchronous circuit with clock period $\Phi\left(G_{r}\right) \leq c$, if such a retiming exists.

1. For each vertex $v \in V$, set $r(v) \leftarrow 0$.

2. Repeat the following $|V|-1$ times:

2.1. Compute graph $G_{r}$ with the existing values for $r$.

2.2. Run Algorithm $\mathrm{CP}$ on the graph $G_{r}$ to determine $\Delta(v)$ for each vertex $v \in V$.

2.3. For each $v$ such that $\Delta(v)>c$, set $r(v) \leftarrow r(v)+1$. 
3. Run Algorithm CP on the circuit $G_{r}$. If $\Phi\left(G_{r}\right)>c$, then no feasible retiming exists. Otherwise, $r$ is the desired retiming.

Proof of Correctness. ${ }^{8}$ Algorithm FEAS works by relaxation. Step 1 specifies an initial tentative retiming in which each vertex has zero lag (so that $G_{r}=G$ ). Each iteration of step 2 is equivalent to one pass of a Bellman-Ford algorithm on the constraints in Theorem 7 . We assume that the tentative values produced during each pass over the constraint set depend only on the tentative values from the previous pass.

After each iteration of step 2, the tentative retiming is guaranteed to be legal. Consider an edge $u \stackrel{e}{\rightarrow} \mathrm{v}$ in $G_{r}$. If the retimed weight $w_{r}(e)$ is strictly positive at the beginning of the iteration, then it will be nonnegative at the end of the iteration because $r(u)$ can increase by at most 1 and $r(v)$ cannot decrease. If $w_{r}(e)=0$ at the beginning of the iteration and if $r(u)$ is incremented, then $r(v)$ will be incremented as well because $\Delta(v) \geq \Delta(u)+d(u) \geq \Delta(u)>c$ in this case.

It remains to show that step 2 simulates a pass of a Bellman-Ford algorithm on the constraints from Theorem 7 . Since the tentative retiming is always legal at the beginning of an iteration, the constraints 7.1 are already satisfied. Thus, the relaxation step in the inner loop of the Bellman-Ford algorithm does not change the value of any $r(v)$ for these constraints.

To see that the effects of the relaxations due to the constraints 7.2 are achieved, consider any two vertices $u, v \in V$. If we have $D(u, v) \leq c$, then no inequality for critical paths from $u$ to $v$ occurs in this constraint set. If the retimed critical path weight $W_{r}(u, v)=W(u, v)+r(v)-r(u)$ is positive, then the corresponding inequality in the constraint set is already satisfied. Finally, when $D(u, v)>c$ and $W(u, v)=0$, there is some path $u \stackrel{p}{\rightarrow} v$ such that $w_{r}(p)=W_{r}(u, v)=0$ and $d(p)=$ $D(u, v)$. The existence of this path implies that $\Delta(v) \geq d(p)=D(u, v)>c$, so that $r(v)$ will be given the new value $r(v)+1=r(u)+W_{r}(u, v)-W(u, v)+1=r(u)-$ $W(u, v)+1$, precisely achieving the effect of the desired relaxation of the constraint $r(u)-r(v) \leq W(u, v)-1$. Conversely, $r(v)$ is incremented only when there exists some path $x \stackrel{p}{\rightarrow} v$ such that $w_{r}(p)=0$ and $d(p)=\Delta(v)>c$, implying that $D(x, v) \geq$ $d(p)>c$ and $W_{r}(u, v)=0$.

Algorithm FEAS can be used to improve the clock-period-minimization algorithm OPT1.

Algorithm OPT2 (clock-period minimization). Given a synchronous circuit $G=$ $\langle V, E, d, w\rangle$, this algorithm determines a retiming $r$ such that $\Phi\left(G_{r}\right)$ is as small as possible.

Run Algorithm OPT1 using Algorithm FEAS in step 3, rather than the BellmanFord algorithm, to test whether each potential clock period $c$ is feasible.

Algorithm OPT2 runs in $O(|V||E| \lg |V|)$ time.

${ }^{8} \mathrm{~A}$ more detailed proof can be found in [20]. 
6. A Mathematical-Programming Framework for Retiming. In this section we describe another algorithm for clock-period minimization based on a special case of mixed-integer linear programming. Specifically, we show that the feasible clockperiod test can be performed in $O(|V||E| \lg |V|)$ time. Although this bound is not an improvement over the $O(|V||E|)$ bound for Algorithm FEAS, the mathematical-programming framework in this section provides further insight into retiming.

The feasible clock-period test can be reduced to the following mixed-integer programming problem.

Problem MILP. Let $S$ be a set of $m$ linear inequalities of the form $x_{j}-x_{i} \leq a_{i j}$ on the unknowns $x_{1}, x_{2}, \ldots, x_{n}$, where the $a_{i j}$ are given real constants, and let $k$ be given. Determine feasible values for the unknowns $x_{i}$ subject to the constraint that $x_{i}$ is integer for $i=1,2, \ldots, k$ and real for $i=k+1, k+2, \ldots, n$, or determine that no such values exist.

Although mixed-integer programming is in general NP-complete (because integer programming is $[4$, p. 245]), this special case can be solved in $O(m n+k m \lg n)$ time [15]. The reduction of the feasible clock-period test to Problem MILP makes use of the following lemma.

LEMMA 8. Let $G=\langle V, E, d, w\rangle$ be a synchronous circuit, and let c be a positive real number. Then the clock period $\Phi(G)$ is less than or equal to $c$ if and only if there exists a function $s: V \rightarrow[0, c]$ such that $s(v) \geq d(v)$ for every vertex $v$ and such that $s(v) \geq s(u)+d(v)$ for every zero-weight edge $u \stackrel{e}{\rightarrow} \mathrm{v}$.

Proof. For each vertex $v$, let $\Delta(v)$ be the maximal sum of the combinational delays along any zero-weight path that ends at $v$. (This $\Delta$ is the same as the one in Algorithm CP.) By definition, we have $\Phi(G) \leq c$ if and only if $\Delta(v) \leq c$ for all $v$. If we have $\Phi(G) \leq c$, the function $\Delta$ satisfies the desired properties for $s$. Conversely, if a function $s$ exists that has the desired properties, then we have $\Delta(v) \leq s(v) \leq c$ for every vertex $v$.

Lemma 8 and Corollary 3 together give a characterization of when it is possible to retime a circuit so that the retimed circuit has a clock period of $c$ or less.

LEMMA 9. Let $G=\langle V, E, d, w\rangle$ be a synchronous circuit, and let $c$ be a positive real number. Then there exists a retiming $r$ of $G$ such that $\Phi\left(G_{r}\right) \leq c$ if and only if there exists an assignment of a real value $s(v)$ and an integer value $r(v)$ to each vertex $v \in V$ such that the following conditions are satisfied:

9.1. $-s(v) \leq-d(v)$ for every vertex $v \in V$,

9.2. $s(v) \leq c$ for every vertex $v \in V$,

9.3. $r(u)-r(v) \leq w(e)$ wherever $u \stackrel{e}{\rightarrow} v$, and

9.4. $s(u)-s(v) \leq-d(v)$ wherever $u \stackrel{e}{\rightarrow} v$ such that $r(u)-r(v)=w(e)$. 
Proof. Condition 9.3 captures the requirements for $r$ to be a legal retiming, as given in Corollary 3, namely that $r$ be a mapping from $V$ to $\mathbf{Z}$ such that $G_{r}$ satisfies condition W1 (no negative-weight edges). Conditions 9.1, 9.2, and 9.4 capture the requirement for $G_{r}$ to have a clock period $\Phi\left(G_{r}\right) \leq c$ as given in Lemma 8. (Recall that $G_{r}$ is defined to have $w_{r}(e)=w(e)+r(v)-r(u)$ for each edge $u \stackrel{e}{\rightarrow} \mathrm{v}$.)

Unfortunately, this result does not quite allow us to recast an instance of the feasible clock-period test as an instance of Problem MILP because of the qualifying clause "such that $r(u)-r(v)=w(e)$ " in condition 9.4. The next theorem shows that the conditions can be expressed without such a clause.

THEOREM 10. Let $G=\langle V, E, d, w\rangle$ be a synchronous circuit, and let $c$ be a positive real number. Then there is a retiming $r$ of $G$ such that $\Phi\left(G_{r}\right) \leq c$ if and only if there exists an assignment of a real value $R(v)$ and an integer value $r(v)$ to each vertex $v \in V$ such that the following conditions are satisfied:

10.1. $r(v)-R(v) \leq-d(v) / c$ for every vertex $v \in V$,

10.2. $R(v)-r(v) \leq 1$ for every vertex $v \in V$,

10.3. $r(u)-r(v) \leq w(e)$ wherever $u \stackrel{e}{\rightarrow} v$, and

10.4. $R(u)-R(v) \leq w(e)-d(v) / c$ wherever $u \stackrel{e}{\rightarrow} v$.

Proof. Any solution to the conditions in Lemma 9 can be converted to a solution to the conditions above by using the same values for the $r(v)$ and taking $R(v)=r(v)+s(v) / c$ for each vertex $v$. Conversely, any solution to the conditions above yields a solution to the conditions in Lemma 9 using the substitution $s(v)=c(R(v)-r(v))$

Theorem 10 is the basis for the following improvement on Algorithm OPT1.

Algorithm OPT3 (clock-period minimization). Given a synchronous circuit $G=$ $\langle V, E, d, w\rangle$, this algorithm determines a retiming $r$ such that $\Phi\left(G_{r}\right)$ is as small as possible.

Run Algorithm OPT1, but in step 3, test whether each potential clock period $c$ is feasible in the following manner.

3.1. Use Theorem 10 to produce an instance of Problem MILP that has a solution if and only if clock period $c$ is feasible.

3.2. Use the algorithm from [15] to determine whether the instance of Problem MILP has a solution.

This algorithm can be made to run in $O\left(|V||E| \lg |V|+|V|^{2} \lg ^{2}|V|\right)$ time by choosing efficient algorithms for each of the steps. If Johnson's all-pairs shortestpaths algorithm [7] using the Fibonacci heap data structure due to Fredman and Tarjan [2] is used in Algorithm WD, step 1 runs in $O\left(|V||E|+|V|^{2} \lg |V|\right)$ time. Since there are only $O\left(|V|^{2}\right)$ elements in the range of $D$, step 2 runs in $O\left(|V|^{2} \lg |V|\right)$ time. Each iteration of the binary search in step 3 requires solving an instance of 
Problem MILP with $|V|$ integer variables, $|V|$ real variables, and $2|V|+2|E|=$ $O(|E|)$ inequalities. Thus the total time for step 3 is $O\left(|V||E| \lg |V|+|V|^{2} \lg ^{2}|V|\right)$. The optimal retiming from step 4 is produced as a side effect of step 3.

7. Unit Propagation Delay, Systolic Circuits, and Slowdown. This section examines circuits in which the propagation delays of all functional elements are equal. For such circuits, the clock-period-minimization problem can be solved more simply than for arbitrary circuits. In this section we explore the relation of this class of circuits to systolic computation [8], [9], [11], [14]. We observe that many systolic circuits in the literature can support several independent, interleaved computations. In [14] we introduced a transformation called slowdown which, when coupled with retiming, can be used to produce a systolic circuit from an arbitrary synchronous circuit. In this section we give an efficient algorithm for determining whether any given circuit $G$ can be produced from another circuit (called a reduced form of $G$ ) by slowdown and retiming. If such a reduced circuit exists, then our algorithm finds one.

We define a circuit $G=\langle V, E, d, w\rangle$ to be a unit-delay circuit if each vertex $v \in V$ has propagation delay $d(v)=1$. The next theorem gives a characterization of when a unit-delay circuit has clock period less than or equal to $c$. The theorem is phrased in terms of the graph $G-1 / c$, which is defined as $G-1 / c=\left\langle V, E, d, w^{\prime}\right\rangle$ where $w^{\prime}(e)=w(e)-1 / c$ for every edge $e \in E$. Thus $G-1 / c$ is the graph obtained from $G$ by subtracting $1 / c$ from the weight of each edge in $G$.

THEOREM 11. Let $G=\langle V, E, d, w\rangle$ be a unit-delay synchronous circuit, and let $c$ be any positive integer. Then there is a retiming $r$ of $G$ such that $\Phi\left(G_{r}\right) \leq c$ if and only if $G-1 / c$ contains no cycles having negative weight.

Proof. First, suppose $G-1 / c$ has no negative-weight cycles. We produce a retiming $r$ of $G$ such that $\Phi\left(G_{r}\right) \leq c$. Assume without loss of generality that there is a path from each vertex $v$ of $G$ to some vertex $v_{0}$ (if not, add edges of the form $v \rightarrow v_{0}$ with sufficiently large weight so that no negative-weight cycles are introduced into $G-1 / c)$, and let $g(v)$ be the weight of the shortest path from $v$ to $v_{0}$ in $G-1 / c$. For each vertex $v$, let $r(v)=\lceil g(v)\rceil$.

We now prove that the function $r$ so defined is a legal retiming and that $\Phi\left(G_{r}\right) \leq c$. First we show legality by showing $w_{r}(e)=w(e)+r(v)-r(u) \geq 0$ for every edge $u \stackrel{e}{\rightarrow} v$. The shortest path in $G-1 / c$ from $u$ to $v_{0}$ is at least as short as the path $u \stackrel{e}{\rightarrow} v \stackrel{p}{\rightarrow} v_{0}$, where $p$ is the shortest path (in $G-1 / c$ ) from $v$ to $v_{0}$. Thus, we have $g(u) \leq g(v)+w(e)-1 / c$. Taking ceilings of both sides gives $r(u) \leq$ $\lceil g(v)+w(e)-1 / c\rceil \leq\lceil g(v)\rceil+w(e) \leq r(v)+w(e)$, and thus $w_{r}(e)=w(e)+r(v)-$ $r(u) \geq 0$, as desired.

Next, we must show that the clock period of the retimed circuit $G_{r}$ is at most $c$. That is, we must show that $w_{r}(p) \geq 1$ for any path $u \stackrel{p}{\rightarrow} v$ containing $c$ or more edges. The shortest path from $u$ to $v_{0}$ is at least as short as the path $u \stackrel{p}{\rightarrow} v \stackrel{q}{\rightarrow} v_{0}$, where $q$ is the shortest path from $v$ to $v_{0}$. Furthermore, the total weight along $p$ in 
$G-1 / c$ is at most $w(p)-1$, since there are at least $c$ edges in the path. Thus, we have $g(u) \leq g(v)+w(p)-1$, and $w_{r}(p)=w(p)+\lceil g(v)\rceil-\lceil g(u)\rceil \geq 1$.

On the other hand, suppose $G-1 / c$ contains some cycle $p$ with negative weight. We must prove that $G$ cannot be retimed to have a clock period of $c$ or less. Let $n$ be the number of edges in the cycle $p$. By the definition of $G-1 / c$, we have $w(p)-n / c=w^{\prime}(p)$, where $w^{\prime}$ is the edge-weight function for $G-1 / c$. But by supposition, $w^{\prime}(p)$ is negative, which means that $w(p)-n / c<0$, that is, the cycle $p$ contains fewer than $n / c$ registers in $G$. But retiming leaves the number of registers on any cycle unchanged (Corollary 2). Thus, no matter how the fewer than $n / c$ registers are distributed on the cycle of $n$ vertices, there must be some register-free path with at least $c$ edges and, therefore, with at least $c+1$ vertices. Consequently, $G$ cannot be retimed to have a clock period of $c$ or less.

To test whether there is a retiming $r$ of a unit-delay circuit $G$ such that $\Phi\left(G_{r}\right) \leq c$, we can use the Bellman-Ford algorithm to find the weight $g(v)$ of the shortest path in $G-1 / c$ from each vertex $v$ to an arbitrary vertex $v_{0}$. If the shortest-path weights are not well defined, the Bellman-Ford algorithm detects a negative-weight cycle, which means that no retiming exists. Thus, the feasible clock-period test can be performed in $O(|V||E|)$ time for unit-delay circuits using the Bellman-Ford algorithm directly.

A systolic circuit is a unit-delay circuit in which there is at least one register along every interconnection between two functional elements. Thus the clock period of a systolic circuit is the minimum possible-the propagation delay through a single functional element. Systolic circuits have been studied extensively [8], [9], [11], [14], and they have many applications including signal processing, matrix manipulation, machine vision, and raster graphics.

Interpreted in the context of systolic circuits, Theorem 11 is a generalization of the Systolic Conversion Theorem from [14], which says that $G$ can be retimed to be systolic if the constraint graph $G-1$ has no cycles of negative weight. (Simply restrict Theorem 11 to the case where $c=1$.) The Systolic Conversion Theorem is generalized in a different way in [14], however, through the idea of slowdown.

For any circuit $G=\langle V, E, d, w\rangle$ and any positive integer $c$, the circuit $c G$ is the circuit obtained by multiplying all the register counts in $G$ by $c$. That is, the circuit $c G$ is defined as $c G=\left\langle V, E, d, w^{\prime}\right\rangle$ where $w^{\prime}(e)=c w(e)$ for every edge $e \in E$. All the data flow in $c G$ is slowed down by a factor of $c$, so that $c G$ performs the same computations as $G$, but takes $c$ times as many clock ticks and communicates with the external interfaces only on every $c$ th clock tick. In fact, $c G$ acts as a set of $c$ independent, interleaved instances of $G$.

If a circuit $G$ can be obtained by retiming a circuit of the form $c G^{\prime}$, then we say that $G$ is a $c$-slow circuit, and, more specifically, that $G$ is a $c$-slow form of $G^{\prime}$. In this situation, we say $G^{\prime}$ is a reduced form of $G$. The main advantage of a $c$-slow circuit is that it can often be retimed to have a shorter clock period than any of its reduced forms. For some applications, throughput is the issue, and multiple, interleaved streams of computation can be effectively utilized. A $c$-slow circuit that is systolic offers maximum throughput. 
The following corollary to Theorem 11 tells when a circuit has a $c$-slow form which is systolic.

COROLlaRY 12. Let $G=\langle V, E, d, w\rangle$ be a unit-delay synchronous circuit, and let $c$ be an arbitrary positive integer. Then the following are equivalent:

12.1 The graph $G-1 / c$ has no negative-weight cycles.

12.2. The circuit $G$ can be retimed to have clock period less than or equal to $c$.

12.3. The circuit $c G$ can be retimed to be systolic.

ProOF. That 12.1 and 12.2 are equivalent is exactly Theorem 11 . The equivalence of 12.1 and 12.3 follows by applying Theorem 11 to $c G$ with clock period 1 , and observing that $c G-1$ has a negative-weight cycle if and only if $G-1 / c$ has negative-weight cycle.

The registers of a circuit of the form $c G$ are naturally divided into $c$ equivalence classes. Given any two registers $A$ and $B$ in $c G$, the number of registers on any two paths from register $A$ to register $B$ are congruent modulo $c$. Moreover, if we consider undirected paths, in which edges can be traversed in the reverse direction, and if we generalize the notion of path weight by adding 1 for each register on a forward edge and subtracting 1 for each register on a reverse edge, the register counts of two undirected paths from register $A$ to register $B$ are also congruent modulo $c$. Consequently, the registers are naturally divided into equivalence classes according to their undirected path weight (modulo $c$ ) from an arbitrary vertex.

At any given time step, any two registers in different equivalence classes contain data from independent streams of computation-data that can never arrive at inputs of the same functional element at the same time. Although retiming destroys the individual identities of the registers, Lemma 1 guarantees that the registers of any $c$-slow circuit can still be partitioned into $c$ such equivalence classes.

Using the notion of equivalence classes of registers, the following scenario illustrates the relationships given in Corollary 12 . Let $G=\langle V, E, d, w\rangle$ be a unit-delay synchronous circuit. Find an integer $c$ such that $G-1 / c$ has no negative-weight cycles, and consider the circuit $c G$. There is a retiming $r$ of $c G$ such that $(c G)_{r}$ is systolic. If we remove all the registers in the $c$-slow circuit $(c G)_{r}$ except for those in one equivalence class, the resulting circuit is a retimed form of the original circuit $G$, and its clock period is less than or equal to $c$.

Many systolic circuits appearing in the literature are 2-slow or 3-slow-even if the ideas of slowdown and retiming were not explicitly used in their design. For example, the systolic algorithms for band-matrix multiplication and $L U$-decomposition from [9] are 3-slow and can support three independent, interleaved streams of computation. If all independent streams of computation cannot be utilized in a $c$-slow circuit, it may be desirable to remove all registers except for those in one equivalence class. The following algorithm determines if a circuit is actually a $c$ slow form of another, and if so, produces a reduced form of the circuit. 
Algorithm R (remove all but one equivalence class of registers in a circuit). Given a synchronous circuit $G=\langle V, E, d, w\rangle$ this algorithm determines the largest $c$ such that $G$ is $c$-slow and produces a reduced circuit $G^{\prime}$ such that $G$ is a $c$-slow form of $G^{\prime}$.

1. For each vertex $v \in V$, set $\operatorname{dist}(v)$ to the weight of some undirected path from $v$ to an arbitrary vertex $v_{0} \in V$.

2. Compute $c=\operatorname{gcd}\{w(e)+\operatorname{dist}(v)-\operatorname{dist}(u): u \stackrel{e}{\rightarrow} v\}$.

3. For each vertex $v \in V$, set $r(v)=\operatorname{dist}(v) \bmod c$.

4. Produce $G^{\prime}=\left\langle V, E, d, w^{\prime}\right\rangle$, where $w^{\prime}(e)=(w(e)+r(v)-r(u)) / c$ for each edge $u \stackrel{e}{\rightarrow} v$.

Proof of Correctness. We first show that for each edge $u \stackrel{e}{\rightarrow} v$, the value $w^{\prime}(e)$ is a legal register count. By construction, $c$ evenly divides $w(e)+r(v)-r(u)$ because $c$ divides $w(e)+\operatorname{dist}(v)-\operatorname{dist}(u) .^{9}$ Thus, for any edge, the register count $w^{\prime}(e)$ produced in step 4 is guaranteed to be an integer. In addition, $w^{\prime}(e)$ is guaranteed to be strictly greater than -1 beause $r(u)$ must be less than $c$ and $w(e)+r(v)$ is at least 0 . Since we have just shown that $w^{\prime}(e)$ is an integer, it must be nonnegative.

The construction in step 4 directly provides the identity $G^{\prime}=G_{r} / c$, and thus $G$ is a $c$-slow form of $G^{\prime}$. We show that the $c$ computed in step 2 is the largest possible. Suppose there is a $c^{\prime}$ such that $G$ is a $c^{\prime}$-slow form of another circuit $G^{\prime}$. We wish to show that $c^{\prime}$ divides $w(e)+\operatorname{dist}(v)-\operatorname{dist}(u)$ for each edge $u \stackrel{e}{\rightarrow} \mathrm{v}$, and thus that $c^{\prime}$ divides $c$. For every vertex $v$, the weights of all undirected paths in $c^{\prime} G^{\prime}$ from $v$ to $v_{0}$ are congruent modulo $c^{\prime}$. Since retiming changes all path weights between two vertices by the same amount (which is provable for undirected paths by generalizing Lemma 1), it must be the case that in $G$, the weights of all undirected paths from $v$ to $v_{0}$ are congruent modulo $c^{\prime}$. In particular, the weight of the path $u \rightarrow v_{0}$ that determines $\operatorname{dist}(u)$ and the weight $w(e)+\operatorname{dist}(v)$ of the path $u \stackrel{e}{\rightarrow} \mathrm{v} \rightarrow v_{0}$ must be congruent modulo $c^{\prime}$. Hence $c^{\prime}$ divides $w(e)+\operatorname{dist}(v)-\operatorname{dist}(u)$.

Step 1 of Algorithm R can be performed in time $O(|V|+|E|)=O(|E|)$ by depth-first search. Step 3 runs in $O(|V|)$ time, and step 4 takes $O(|E|)$ time. Step 2 takes more work, but not much more. The computation of the greatest common divisor (gcd) of $|E|$ integers can be performed in $O(|E|+\lg x)$ time, where $x$ is the least nonzero absolute value of any of the numbers. Just start with this value $x$-which can be found in $O(|E|)$ time-as a tentative gcd, and gcd in each of the other numbers in any order. Each mod operation in Euclid's algorithm either uses up one of the $|E|$ numbers, or else divides the current tentative gcd by the golden ratio $(1+\sqrt{5}) / 2 .{ }^{10}$ (As a practical matter, starting with any of the $|E|$ numbers as the initial tentative gcd would give reasonable performance, since the number of

\footnotetext{
${ }^{9}$ If $w(e)+\operatorname{dist}(v)-\operatorname{dist}(u)=0$ for every edge $u \stackrel{e}{\rightarrow} v$, then in Step 2 we get $c=\infty$. Using the standard convention that $x \bmod \infty=x$ and $x / \infty=0$, Algorithm $\mathrm{R}$ yields a reduced graph $G^{\prime}$ in which each edge $u \stackrel{e}{\rightarrow} v$ has weight $w^{\prime}(e)=0$.

${ }^{10}$ That is, if $n$ mod operations are performed to compute a new tentative gcd, then it will be smaller than the old tentative ged by at least a factor of $((1+\sqrt{5}) / 2)^{n-1}$.
} 
registers in a typical circuit is much less than exponential in the number of edges.) Thus the total running time of Algorithm $\mathrm{R}$ is $O(|E|+\lg x)$.

Observe that Algorithm R works not only for unit-delay circuits, but for any synchronous circuit. Furthermore, when the extra equivalence classes of registers from a $c$-slow circuit are removed, the clock period of the reduced circuit is not unduly lengthened. The definition of $w^{\prime}$ in step 4 provides that, for any path $p$ of weight $w(p) \geq c$ in $G$, we have $w^{\prime}(p)>0$, which implies that $\Phi\left(G^{\prime}\right) \leq c \Phi(G)$. To guarantee the minimum clock period, however, the reduced circuit must generally be retimed.

A systolic circuit that is naturally $c$-slow can be converted by Algorithm R into a circuit that performs an operation on every clock tick and whose clock period is bounded by $c$. This conversion can result in a performance advantage because, in practice, there are time penalties associated with the loading of registers. Because of this overhead, $c$ clock ticks of a circuit with nominal period 1 typically use more time than one clock tick of a circuit with nominal period $c$. Also, a reduction in registers may save chip area, which can lead to further performance improvements since the wires will in general be shorter. A possible disadvantage of reducing the number of equivalence classes of registers is that throughput is also reduced in cases where the independent streams of computation might be effectively utilized.

8. Register Minimization and Fanout. Thus far we have concentrated on clock period as the objective function for determining a retiming. In Section 7, however, we showed that the number of registers in a circuit could sometimes be reduced by a method other than retiming. This section shows that the problem of retiming a circuit to minimize the total state of a circuit is polynomial-time solvable by reducing that problem to a minimum-cost flow $[10$, p. 129] problem. We also show that the total state of a circuit can be minimized subject to a bound on the clock period. These results can be extended to reflect the widths of the interconnections and ways by which fanout is modeled.

For a given circuit $G=\langle V, E, d, w\rangle$, the state-minimization problem is to determine a retiming $r$ such that the total state $S\left(G_{r}\right)=\Sigma_{e \in E} w_{r}(e)$ of the retimed circuit is minimized. This problem can be solved in polynomial time as the following theorem shows.

THEOREM 13. The state-minimization problem can be reduced to the minimum-cost flow problem.

Sketch of Proof. Let $G=\langle V, E, d, w\rangle$ be a circuit. We seek a retiming $r$ such that the total state $S\left(G_{r}\right)=\Sigma_{e \in E} w_{r}(e)$ of the retimed circuit is minimized. By the definition of $w_{r}$, we have

$$
\begin{aligned}
S\left(G_{r}\right) & =\sum_{e \in E} w_{r}(e) \\
& =\sum_{u^{\underline{e} v}}(w(e)+r(v)-r(u)) \\
& =S(G)+\sum_{v \in V} r(v)(\text { indegree }(v)-\operatorname{outdegree}(v)) .
\end{aligned}
$$


Since $S(G)$ is constant, minimizing $S_{r}(G)$ is equivalent to minimizing the quantity

$$
\sum_{v \in V} r(v)(\text { indegree }(v) \text { - outdegree }(v))
$$

which is a linear combination of the $r(v)$ since (indegree $(v)$-outdegree $(v)$ ) is constant for each $v$. The minimization is subject to the constraint that, for each edge $u \stackrel{e}{\rightarrow} \mathrm{v}$, the register count $w_{r}(e)$ is nonnegative-that is,

$$
r(u)-r(v) \leq w(e) .
$$

We can regard each edge of $e \in E$ as a network flow arc having infinite capacity and having cost $w(e)$ per unit of flow. The dual of the linear-programming problem given by (5) and (6) asks that we assign to each edge $e$ a nonnegative flow $f(e)$ such that the net flow out of any vertex $v$ is

$$
\sum_{v \rho ?} f(e)-\sum_{? \hookrightarrow_{v}} f(e)=\text { outdegree }(v)-\text { indegree }(v),
$$

and such that the total cost $\sum_{e \in E} w(e) f(e)$ is minimized. This problem can be expressed directly as a minimum-cost flow problem by augmenting the flow graph with a source and a sink, each of which is connected to each vertex by an edge whose capacity is determined by (7). The lags $r(v)$ in the minimum-state retiming are the dual variables (potentials) for the optimal flow $f^{*}(e)$, which most minimum-cost flow algorithms compute.

The dominant cost in solving the state-minimization problem is solving the minimum-cost flow problem, for which many algorithms exist [1], [3], [5], [6], [18]. Using the algorithm due to Orlin [18], for example, the state-minimization problem can be solved in $O\left(|E|^{2} \lg |V|+|V||E| \lg ^{2}|V|\right)$ time. Under the assumption that the largest number of registers on any single edge in the circuit is at most polynomial in $|V|$, we can use the algorithm due to Goldberg and Tarjan [6] to solve the state-minimization problem in $O\left(|V||E| \lg |V| \lg \left(|V|^{2} /|E|\right)\right)$ time.

More complicated problems can be solved within the same framework. For example, the total state of a circuit can be minimized subject to a bound on the clock period. Given a maximum allowable clock period $c$, we wish to find a retiming $r$ that minimizes the state $S\left(G_{r}\right)$ of the retimed circuit subject to the condition that $\Phi\left(G_{r}\right) \leq c$. In this case, we must minimize the quantity (5) subject to the constraints from Theorem 7 , which require that $r(u)-r(v) \leq w(e)$ whenever $u \stackrel{e}{\rightarrow} v$, and that $r(u)-r(v) \leq W(u, v)-1$ whenever $D(u, v)>c$. The state minimization problem remains the dual of a minimum-cost flow problem, but the flow graph is augmented with additional edges. ${ }^{11}$

The state-minimization problem can be generalized by allowing registers on different edges to have different costs. For example, it may be cheaper to add a

\footnotetext{
${ }^{11}$ See Section VII.2 of [20], for a more extended discussion of the minimum-cost flow duals of the variants of the state minimization problems discussed in this section.
} 
register along a one-bit wide control path than along a 32-bit wide data path. We may model such situations by assigning to each edge $e$ a breadth $\beta(e)$ proportional to the cost of adding a register along $e$. The objective function which we must minimize is then given by

$$
\sum_{v \in V} r(v)\left(\sum_{? \sum_{v}} \beta(e)-\sum_{v \in ?} \beta(e)\right)
$$

and the constraints on the $r(v)$ are unchanged. This problem is still the dual of a minimum-cost flow problem since the quantity in the large parentheses is a constant for each $v$. Although the $\beta(e)$ need not be integers, if there is a solution to the state-minimization problem, there is an integer optimal solution because the linear-programming tableau for the problem is unimodular and the right-hand side is an integer vector.

In a physical circuit, a signal from a register or functional element may fan out to several functional elements. As was mentioned in footnote 5 in Section 2, we model this situation with several different edges in the circuit graph. For the clock-periodminimization problem, there was no harm in modeling fanout in this manner, but for the state-minimization problem, there can be. The difficulty that arises in the state-minimization problem is that registers can be shared along the physical interconnection. The objective functions (5) and (8) do not take sharing into account.

Fanout can be incorporated into the model in several ways that allow the sharing of registers to be accounted for exactly. We begin by looking at the situation in Figure 5(a) where one vertex $u$ has an output that fans out to two vertices $v_{1}$ and $v_{2}$. To deal properly with this situation in the state-minimization problem, it is sufficient to introduce a dummy vertex $\hat{u}$ with zero propagation delay which models the fork of the interconnection, as is shown in Figure 5(b). When the

(a)

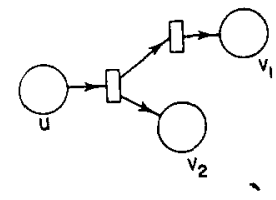

(b)

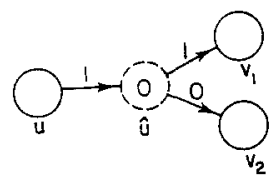

(c)

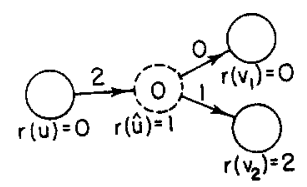

Fig. 5. Modeling two-way fanout with an extra vertex having delay zero. 
circuit is retimed to minimize the number of registers, either the edge from $\hat{u}$ to $v_{1}$ or the edge from $\hat{u}$ to $v_{2}$ will have zero register count, and the edge from $u$ to $\hat{u}$ will have the shared registers. In Figure 5(c) the edge to $v_{1}$ ends up with zero weight after retiming so as to minimize the total number of registers.

Large multiway forks present some modeling alternatives not encountered in the two-way case. If a physical interconnection is to be modeled, the fork can be decomposed into several two-way forks. (In fact, our concern for modeling the physical interconnection prompted us to design Correlator 1 with the $x_{i}$ running through the comparators rather than with multiway fanout directly from the external interface.)

For logical design, however, it may be undesirable to model the physical interconnection. In the case of a three-way fork, for instance, we might wish to share the largest possible number of registers between the two edges with greatest register counts, regardless of which two edges these end up being. Modeling a $k$ way fork for $k \geq 3$ by decomposing the interconnection into two-way forks will not work.

A solution to this problem of modeling $k$-way fanout with maximum register sharing is depicted in Figure 6. An output of vertex $u$, having breadth $\beta$, fans out to $v_{1}, \ldots, v_{k}$ along edges $u \stackrel{e_{1}}{\rightarrow} v_{1}, u \stackrel{e_{2}}{\rightarrow} v_{2}, \ldots, u \stackrel{e_{k}}{\rightarrow} v_{k}$. In the retimed circuit, the cost of this fanout should be $\beta$ times the maximum of retimed edge weights $w_{r}\left(e_{i}\right)$. So that the register count cost function $S\left(G_{r}\right)$ will properly model the register sharing, we first add a dummy vertex $\hat{u}$ with zero propagation delay. Letting $w_{\max }=$ $\max _{1 \leq i \leq k} w\left(e_{i}\right)$, we add edges $v_{i} \stackrel{e_{i}}{\rightarrow} \hat{u}_{1}$ with weights $w\left(\hat{e}_{i}\right)=w_{\max }-w\left(e_{i}\right)$. Finally, we give all edges $e_{i}$ and $\hat{e}_{i}$ breadths of $\beta / k$.

The modified circuit graph accurately models the sharing of registers among the edges $e_{i}$ involved in the fanout when the state is minimized. For any retiming $r$, Lemma 1 dictates that the weights $w_{r}\left(p_{i}\right)$ of all paths $p_{i}=u \stackrel{e_{i}}{\rightarrow} v_{i} \stackrel{\hat{e}_{i}}{\rightarrow} \hat{u}$ will be identical since they are identical in the unretimed circuit. The retimed register counts $w_{r}\left(e_{i}\right)$ are constrained by the rest of the circuit, but the weights $w_{r}\left(\hat{e}_{i}\right)$ will be as small as possible because $\hat{u}$ is a sink in the graph. Thus the register count of one
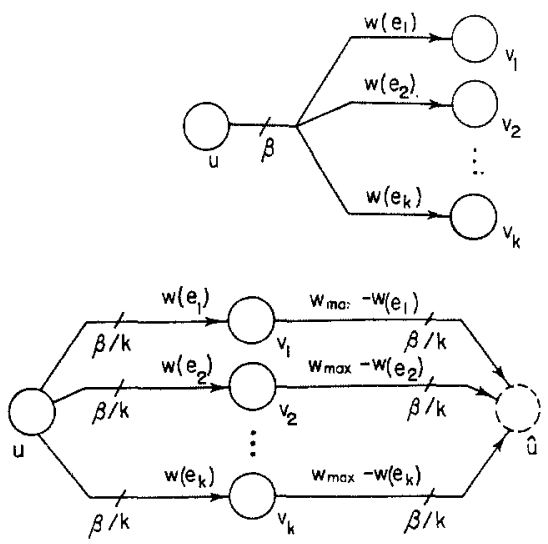

Fig. 6. A gadget for modeling the cost of multiway fanout with maximal sharing of registers. 
of the $\hat{e}_{i}$ will be zero, and therefore the weight of each path $p_{i}$ will be $\max _{1 \leq i \leq k} w_{r}\left(e_{i}\right)$. Since there are $k$ paths, each with breadth $\beta / k$, the total cost of the paths will be $\beta \cdot \max _{1 \leq i \leq k} w_{r}\left(e_{i}\right)$ as desired.

9. A More General Model for Propagation Delay. In this section we extend the methods of Sections 5 and 6 to deal with functional elements in which the propagation delays through individual functional elements are nonuniform. In an adder, for example, the propagation delay from a low-order input bit to a highorder output bit may be far greater than the propagation delay from a low-order input bit to a low-order output bit or from a high-order input bit to a high-order output bit. Thus, the worst-case propagation delay through two cascaded adders can be much less than twice the worst-case propagation delay through a single adder. This section gives a more general circuit model to handle this commonly occurring situation. We show how the retiming problem in this model can be reduced to simple mixed-integer programming as in Section 6. We also give a more efficient relaxation algorithm similar to that in Section 5 .

We may take into account nonuniform propagation delays through functional elements by modifying the model for synchronous circuits given in Section 2, so that from each input to each output of a given functional element, an independent propagation delay may be assigned. Figure 7 shows graphically the "insides" of a functional element in this model. The vertex $v$ contains internal edges drawn from a set $F_{v} \subseteq\{e: ? \stackrel{e}{\rightarrow} v\} \times\{e: v \stackrel{e}{\rightarrow} ?\}$. We denote the set of all internal edges of all vertices as $F=\bigcup_{v \in V} F_{v}$.

We augment our arrow notation as follows. For internal edges $f_{a} \in F_{u}$ and $f_{b} \in F_{v}$, we use the notation $f_{a} \stackrel{e}{\rightarrow} f_{b}$ to indicate that normal edge $e$ connects internal edge $f_{a}$ of $u$ to internal edge $f_{b}$ of $v$. This means not only that $u \stackrel{e}{\rightarrow} v$, but also that there are edges? $\stackrel{e_{1}}{\rightarrow} u$ and $v \stackrel{e_{O}}{\rightarrow}$ ? such that $f_{a}=\left(e_{1}, e\right)$ and $f_{b}=\left(e, e_{0}\right)$. Notice that a single edge $u \stackrel{e}{\rightarrow} v$ typically connects multiple internal edges of $u$ with multiple internal edges of $v$.

The propagation delay function $d$, rather than being a function from $V$ to the nonnegative reals, is a function from $F$ to the nonnegative reals. For an internal edge $f=\left(e_{1}, e_{2}\right) \in F_{v}$, the value $d(f)$ denotes the propagation delay through $f$. A given output of a functional element $v$ need not depend on all the inputs. In an adder, for example, the values of the high-order input bits have no effect on the loworder bits of the output.

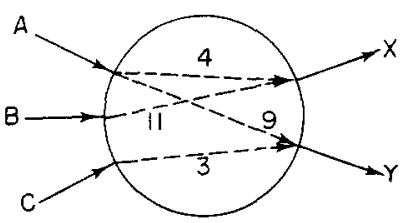

Fig. 7. A functional element with nonuniform propagation delays. The time at which output $X$ must settle is either 4 esec after input $A$ settles or $11 \mathrm{esec}$ after input $B$ settles, whichever is later. 
We view paths in the extended model as going from an internal edge, via an alternating sequence of normal and internal edges, to an internal edge. For any path $p=f_{0} \stackrel{e_{0}}{\rightarrow} f_{1} \stackrel{e_{1}}{\rightarrow} \cdots \stackrel{e_{k-1}}{\rightarrow} f_{k}$, the delay is naturally defined as $d(p)=d\left(f_{0}\right)+$ $d\left(f_{1}\right)+\cdots+d\left(f_{k}\right)$, and the register count of the path is defined as $w(p)=$ $w\left(e_{0}\right)+w\left(e_{1}\right)+\cdots+w\left(e_{k-1}\right)$. We use the notation $u \stackrel{p}{\rightarrow} v$ to denote that path $p$ goes from some internal edge of vertex $u$ to some internal edge of vertex $v$.

The clock period $\Phi(G)$ of a circuit $G=\langle V, E, d, w\rangle$ is the maximum delay along any path of zero weight. We define retiming, which affects $w$ but not $d$, exactly as it is defined in the model of Section $2 .{ }^{12}$

The following results show how the clock-period-minimization problem for a circuit $G$ under the extended model can be reduced to an instance of Problem MILP having one integer variable for each functional element of $G$ and one real variable for each edge of $G$. The results, which parallel Lemma 8, Lemma 9, and Theorem 10, are presented without proof.

LEMMA 14. Let $G=\langle V, E, d, w\rangle$ be a synchronous circuit in the extended model, and let $c$ be a positive real number. Then the clock period $\Phi(G)$ is less than or equal to $c$ if and only if there exists a function $s: E \rightarrow[0, c]$ such that

14.1. $s(e) \geq d(f)$ wherever $f \stackrel{e}{\rightarrow}$ ?, and

14.2. $s\left(e_{b}\right) \geq s\left(e_{a}\right)+d(f)$ wherever $\stackrel{e_{a}}{\rightarrow} f \stackrel{e_{b}}{\rightarrow}$ ? and $w\left(e_{a}\right)>0$.

LEMMA 15. Let $G=\langle V, E, d, w\rangle$ be a synchronous circuit in the extended model, and let $c$ be a positive real number. Then there is a retiming $r$ of $G$ such that $\Phi\left(G_{r}\right) \leq c$ if and only if there exists an assignment of a real value $s(e)$ to each edge $e \in E$ and an integer value $r(v)$ to each vertex $v \in V$ such that the following conditions are satisfied:

$15.1-s(e) \leq-d(f)$ wherever $f \stackrel{e}{\rightarrow}$ ?,

$15.2 s(e) \leq c$ for every edge $e \in E$,

$15.3 r(u)-r(v) \leq w(e)$ wherever $u \stackrel{e}{\rightarrow} v$, and

15.4. $s\left(e_{a}\right)-s\left(e_{b}\right) \leq-d(f)$ wherever $u \stackrel{e_{a}}{\rightarrow} f \stackrel{e_{b}}{\rightarrow}$ ?, $r(u)-r(v)=w\left(e_{a}\right)$, and $f \in F_{v}$.

THEOREM 16. Let $G=\langle V, E, d, w\rangle$ be a synchronous circuit in the extended model, and let $c$ be a positive real number. Then there is a retiming $r$ of $G$ such that $\Phi\left(G_{r}\right) \leq c$ if and only if there exists an assignment of a real value $R(e)$ to each edge $e \in E$ and an integer value $r(v)$ to each vertex $v \in V$ such that the following conditions are satisfied:

16.1. $r(v)-R(e) \leq-d(f) / c$ wherever $f \stackrel{e}{\rightarrow}$ ? and $f \in F_{v}$.

16.2. $R(e)-r(v) \leq 1$ wherever $v \stackrel{e}{\rightarrow}$ ?,

\footnotetext{
12 The possibility that the internal connections between the inputs and outputs of a functional element may not be a complete bipartite graph gives rise to some technical differences between the extended model and the model of Section 2. First, condition W2 need only be imposed for those cycles in which consecutive edges are actually connected by the internal data paths in the vertices. Second, retiming may not be the only way to adjust register counts so that function is guaranteed to be preserved-if the (undirected) graph of internal connections in some functional element is not connected, then the element can be broken up into two or more independent components which can be given different lags.
} 
16.3. $r(u)-r(v) \leq w(e)$ wherever $u \stackrel{e}{\rightarrow} v$, and 16.4. $R\left(e_{a}\right)-R\left(e_{b}\right) \leq w\left(e_{a}\right)-d(f) / c$ wherever $\stackrel{e_{a}}{\rightarrow} f \stackrel{e_{b}}{\rightarrow}$ ?.

Theorem 16 says that the problem of testing whether a given clock period is feasible for a circuit $G=\langle V, E, d, w\rangle$ in the extended model can be efficiently reduced to an instance of Problem MILP having $k=|V|$ integer variables, $n-k=|E|$ real variables, and $m=2|E|+2|d|$ inequalities, where $|d|$ is the number of pairs $\left(e_{a}, e_{b}\right)$ of edges for which $d\left(e_{a}, e_{b}\right)$ is defined. Thus the feasible clock-period test can be performed in $O(|d|(|E|+|V| \lg |E|))$ time.

We can reduce the cost of the feasibility test to $O(|V||F|)$ by using an algorithm similar to Algorithm FEAS. The dominant cost of this computation is due to $|V|$ executions of an algorithm similar to Algorithm $\mathrm{CP}$, each of which runs in $O(|F|)$ time.

The clock-period-minimization algorithm for circuits in the extended model is similar to Algorithm OPT2 which performs a binary search over a set of possible values for the minimal clock period. By the same argument used in Corollary 6 for the model of Section 2, the optimal clock period must be equal to $D(u, v)$ for some pair of vertices $u$ and $v$, where $D$ and $W$ are defined as in (4), but with the semantics of the notation interpreted in the extended model. The values $D(u, v)$ for all connected pairs of vertices $u$ and $v$ can be found in $O(|E||F|+|V||E| \lg |E|)$ time by an algorithm similar to Algorithm WD. The key step is to apply Johnson's allpairs shortest-paths algorithm [7] to the edge-weighted graph $H=\langle E, F, w d\rangle$, where the weighting function is defined by $w d(f)=(w(e),-d(f))$ wherever $\stackrel{e}{\rightarrow} f$ in $G$ (that is, wherever $\stackrel{f}{\rightarrow}$ ? in $H$ ). Using Fibonacci heaps [2] for the priority queue in Johnson's algorithm, a time bound of $O\left(|E||F|+|E|^{2} \lg |E|\right)$ can be achieved. One additional observation is required to prove the claimed time bound of $O(|E||F|+|V||E| \lg |E|)$. The dominant cost of computing $W$ and $D$ by Johnson's all-pair shortest-paths algorithm is due to $|E|$ applications of Dijkstra's algorithm to find shortest paths from each vertex $e \in H$ to each other vertex. Since $W$ and $D$ are defined on $V \times V$ rather than on $E \times E$, however, we really only need to solve $|V|$ problems of the form: Given a vertex $v \in V$, find for each $e \in E$ the weight of $a$ shortest path from $v$ to $e$. Each such problem can be solved by using Dijkstra's algorithm to find the shortest-path weights from a set of vertices in $H$ (namely, from any $x \in E$ such that $? \stackrel{x}{\rightarrow} v$ in $G$ ), rather than from a single vertex. Using Fibonacci heaps, the claimed running time for computing $W$ and $D$ is obtained. The total cost of clock-period minimization in the extended model is therefore $O(|E||F|+|V||E| \lg |E|)+O(|V||F| \lg |V|)=O(|E||F|+|V||F| \lg |V|)$.

10. Concluding Remarks. Our goal has been to provide a general framework for the precise understanding of circuit timing. Through the use of a simple graphtheoretic model, we have been able to cast a variety of circuit timing problems in purely combinatorial terms. We believe our approach to be robust. Many other circuit models and many other circuit problems can be handled within the basic framework. We take time here to discuss a few. 
Pipelining. An important special case of clock-period minimization is the problem of optimally pipelining combinational circuitry. In a combinational circuit, all register counts are zero, and thus the circuit graph is acyclic. We can consider the circuit to have one input interface $v_{\mathrm{I}}$ and one output interface $v_{\mathrm{O}}$. By retiming a combinational circuit $G$, we can produce a pipelined circuit $G_{r}$ which achieves a shorter clock period at the cost of introducing a latency of $r\left(v_{\mathrm{O}}\right)-r\left(v_{\mathrm{I}}\right)$ clock ticks for signals to propagate from the input interface $v_{\mathrm{I}}$ to the output interface $v_{\mathbf{0}}$.

In the optimal pipelining problem, we are given a combinational circuit $G$ and a nonnegative integer $l$ and asked to produce a retimed circuit $G_{r}$ with minimum clock period subject to the constraint that the retimed circuit have latency at most $l$. This problem is just the clock-period-minimization problem with the additional constraint that $r(u)-r(v) \leq l$. This additional constraint can be modeled by augmenting the circuit $G$ with an edge $v_{0} \stackrel{e}{\rightarrow} v_{1}$ having weight $w(e)=l$. Also, the methods of Section 8 can be applied to solve the problem of minimizing the state of pipelined circuitry subject to upper bounds on clock period and latency.

Timing at External Interfaces. An external interface may be forced to meet various timing specifications. For instance, if an external interface has a known time delay between the time at which it receives outputs from the circuit and the time at which it presents inputs, the external vertex can be assigned a propagation delay greater than 0 . By augmenting the set of inequalities specified in Theorem 10 , it is often possible for the optimization algorithms to act subject to other constraints. If data must be available to an interface along some edge $v \stackrel{e}{\rightarrow} v_{0}$ within some time $t$ after each clock tick, for example, we can express this by the inequality

$$
R(v)-r\left(v_{0}\right) \leq \frac{t}{c}+w(e)
$$

where $v_{0}$ is the vertex representing the interface. This constraint is equivalent to saying that we must have $\Delta(v) \leq t$ if the register count $w_{r}(e)$ of edge $e$ is zero in the retimed circuit. Similarly, if data from the interface is not available on an edge $v_{0} \stackrel{e}{\rightarrow} v$ until some time $t$ after each clock tick, this constraint can be expressed by the inequality

$$
r\left(v_{0}\right)-R(v) \leq w(e)-\frac{d(v)}{c}-\frac{t}{c}
$$

Geometric considerations. The optimization methods discussed in this paper can be applied largely independently of geometric considerations because although retiming causes the addition and deletion of registers, it otherwise leaves the functional elements and their pattern of interconnection the same. Thus if a given circuit has an area-efficient layout, chances are that a retimed form of the circuit can be laid out efficiently. In some cases, however, the floorplan of a circuit may limit the number of registers on certain interconnections.

The inequalities that constrain the retimed system can be augmented to express these geometric constraints. For example, to specify an upper bound $k$ on the 
number of registers that can fit along some edge $u \stackrel{e}{\rightarrow} v$, we can impose the constraint

$$
r(v)-r(u) \leq k-w(e)
$$

We can also model a situation in which the first $k$ registers on an edge $u \stackrel{e}{\rightarrow} v$ are relatively cheap and additional registers are more expensive. Add an auxiliary vertex $\hat{u}$ in the middle of edge $e$. Then assign a high cost to registers on the edge $u \rightarrow \hat{u}$ and a low cost to registers on the edge $\hat{u} \rightarrow v$, but constrain $\hat{u} \rightarrow v$ to have at most $k$ registers in the retimed system. Solve the system of constraints as in Section 8. On the other hand, if the first register on a connection is expensive and additional registers are cheap, then it is NP-complete to determine whether a circuit can be retimed to achieve a specified bound on register cost [20, pp. 182-183].

Slowdown. In Section 7 we showed how a $c$-slow circuit, which supports $c$ independent streams of computation, can be reduced to support a single stream of computation by removing registers. The notion of $c$-slow circuitry offers new insight into many circuit designs that are not technically $c$-slow. Consider, for example, a 2-slow circuit in which only one stream of computation is being used. The registers in the circuit fall into two equivalence classes, one of which is idle during each clock period. Using Algorithm $\mathrm{R}$ to remove all the registers in one equivalence class is one way to optimize such a circuit.

Another way to save registers is to modify the functional elements to perform slightly different actions on even and odd time steps so that each physical register plays the roles of two logical registers, one in each equivalence class. A cursory examination of the resulting circuit would not reveal that it is 2-slow according to the circuit model, but it would nevertheless communicate with the host only on every other clock tick. Although this method for saving registers may sometimes be acceptable, the overhead of register multiplexing and the complexity of control suggest that Algorithm $\mathrm{R}$ is a more reasonable alternative. Moreover, when confronted with a circuit that communicates externally only on every other clock tick or a circuit whose functional elements perform different operations on alternate clock ticks, we may suspect that it is really a 2-slow circuit in disguise, and that penetrating the disguise might lead to improved performance and simplification of the control logic.

Data-dependent propagation delays. A major deficiency of the circuit model is its inability to represent combinational logic elements with data-dependent propagation delays. For example, if a multiplier can produce an answer quickly whenever one of its inputs is zero, its propagation delay is data dependent. We would like to take advantage of the shorter delay whenever possible in order to speed a larger computation.

While we are unable to model data dependence in the general case, we can sometimes use the extended circuit model of Section 9 to model partially the effects. As an example, in nMOS circuits [16], the transition of a Boolean signal from 0 to 
1 can take much longer than the transition of the same signal from 1 to 0 . We can model this situation somewhat by representing each wire as two edges in the graph, one representing the value 0 on the wire and the other representing 1 . We choose propagation delays for internal edges of a functional element depending on how 0 or 1 inputs affect the output. Unfortunately, we cannot model how the delays affect the clock period exactly, but upper bounds can be obtained which will, for example, properly model the propagation delay through two cascaded inverters.

There is much more to be understood about clocked circuits. Do powerful combinatorial optimization techniques apply to other timing models such as those involving multiphase clocking disciplines? Can data-dependent propagation delays be handled in a reasonably general setting? Is it possible to solve the stateminimization problem with a polynomial-time algorithm that is simpler than the typical algorithms for solving minimum-cost flow problems? Can hierarchically described circuits be optimally retimed in time proportional to their descriptions? Under what circumstances can optimal retimings of parametrized families of circuits be algorithmically obtained?

Retiming is a transformation that can be used to produce efficient circuits, and we have presented a variety of algorithms for automatically retiming circuits. Of great interest, however, are design methodologies in which retiming is performed by an individual instead of an algorithm, as is done in [11], [12], [14], and [20]. Retiming seems to be a valuable technique which could be incorporated into both circuit compilers and interactive design tools.

Acknowledgments. We would like to offer thanks to many individuals for their contributions to this paper. We wish to give special thanks to Flavio Rose of Digital Equipment Corporation. Flavio was a contributor to the first general algorithm for retiming circuits, which appeared in [13] and [19]. A discussion with Ron Rivest and Rich Zippel of MIT led to our investigation of the stateminimization problem. Ravi Boppana of MIT found and corrected several technical errors in an early version of the paper, and Cynthia M. Hibbard suggested many changes that improved the quality of our exposition. Thanks also go to Dan Gusfield of Yale and Jim Orlin of MIT Sloan School for helpful discussions. The ARPAnet was an invaluable resource in the preparation of this paper.

\section{References}

[1] J. Edmonds and R. M. Karp, Theoretical improvements in algorithmic efficiency for network flow problems, Journal of the Association for Computing Machinery, Vol. 19, No. 2, 1972, pp. 248-264.

[2] M. L. Fredman and R. E. Tarjan, Fibonacci heaps and their uses in improved network optimization algorithms, Proceedings of the 25th Annual Symposium on Foundations of Computer Science, IEEE Computer Society, October 1984, pp. 338-346. 
[3] Z. Galil and É. Tardos, An $O\left(n^{2}(m+n \log n) \log n\right)$ min-cost flow algorithm, Proceedings of the 27th Annual Symposium on Foundations of Computer Science, IEEE Computer Society, October 1986, pp. 1-9.

[4] M. R. Garey and D. S. Johnson, Computers and Intractability, Freeman, San Francisco, 1979.

[5] A. V. Goldberg, Efficient Parallel Algorithms for Sequential and Parallel Computers, Ph.D. dissertion, Department of Electrical Engineering and Computer Science, MIT, February 1987.

[6] A. V. Goldberg and R. E. Tarjan, Finding Minimum-Cost Circulations by Successive Approximation, Technical Memorandum MIT/LCS/TM-33, MIT Laboratory for Computer Science, July 1987.

[7] D. B. Johnson, Efficient algorithms for shortest paths in sparse networks, Journal of the Association for Computing Machinery, Vol. 24, No. 1, January 1977, pp. 1-13.

[8] H. T. Kung, Let's design algorithms for VLSI systems, Proceedings of the Caltech Conference on Very Large Scale Integration, C. L. Seitz, ed., Pasadena, California, January 1979, pp. 55-90.

[9] H. T. Kung and C. E. Leiserson, Systolic arrays (for VLSI), Sparse Matrix Proceedings 1978, I. S. Duff and G. W. Stewart, ed., Society for Industrial and Applied Mathematics, 1979, pp. 256-282. (An earlier version appears in Chapter 8 of [16] under the title, "Algorithms for VLSI processor arrays.")

[10] E. L. Lawler, Combinatorial Optimization: Networks and Matroids, Holt, Rinehart and Winston, New York, 1976.

[11] C. E. Leiserson, Area-Efficient VLSI Computation, Ph.D. dissertation, Department of Computer Science, Carnegie-Mellon University, October 1981. Published in book form by the MIT Press, Cambridge, Massachcusetts, 1983.

[12] C. E. Leiserson, Systolic and semisystolic design, Proceedings of the IEEE International Conference on Computer Design/VLSI in Computers (ICCD '83), Rye, New York, October 1983, pp. 627-632.

[13] C. E. Leiserson, Flavio M. Rose, and James B. Saxe, Optimizing synchronous circuitry by retiming," Proceedings of the 3rd Caltech Conference on Very Large Scale Integration, Randal Bryant, ed., California Institute of Technology, 1983, pp. 87-116. Computer Science Press, Rockville, Maryland.

[14] C. E. Leiserson and J. B. Saxe, Optimizing synchronous systems, Journal of VLSI and Computer Systems, Vol. 1, No. 1, Spring 1983, pp. 41-67.

[15] C. E. Leiserson and James B. Saxe, A mixed-integer programming problem which is efficiently solvable, Journal of Algorithms, Vol. 9, 1988, pp. 114-128. (An earlier version appears in Proceedings of the 21st Annual Allerton Conference on Communication, Control, and Computing, October 1983, pp. 204-213.)

[16] C. A. Mead and L. A. Conway, Introduction to VLSI Systems, Addison-Wesley, Reading, Massachusetts, 1980.

[17] P. Ng, W. Glauert, and R. Kirk, A timing verification system based on extracted MOS/VLSI circuit parameters, 18th Design Automation Conference Proceedings, IEEE, 1981, pp. 288-292.

[18] J. B. Orlin, A faster strongly polynomial minimum cost flow algorithm, Proceedings of the 20th Annual ACM Symposium on Theory of Computing, ACM, May 1988, pp. 377-387.

[19] F. M. Rose, Models for VLSI Circuits, Masters thesis, Department of Electrical Engineering and Computer Science, MIT, March 1982. Also available as MIT VLSI Memo No. 82-114.

[20] J. B. Saxe, Decomposable Searching Problems and Circuit Optimization by Retiming: Two Studies in General Transformations of Computational Structures, Ph.D. dissertation, Department of Computer Science, Carnegie-Mellon University, August 1985.

[21] Texas Instruments Incorporated, The TTL Data Book for Design Engineers, Dallas, Texas, 1976. 